\title{
Identités masculines, expression corporelle et éducation physique en France entre 1967 et 1985
}

\section{Identidades masculinas, expressão corporal e educação física na França entre 1967 e 1985}

\section{Masculine Identities, Body language and Physical Education in France between 1967 et 1985}

\author{
Nicolas Iffrig* \\ Jean Saint-Martin*
}

\begin{abstract}
RÉSUMÉ
Au cœur de la France des années 1970 marquée par une vague de bouleversements idéologiques et culturels, l'expression corporelle constitue une expérience novatrice et unique en Education Physique et Sportive (EPS) face à l'hégémonie du sport en EPS. Par son approche dérégulée, personnelle et émancipatrice, l'expression corporelle marque une rupture dans la gestion du corps des enfants scolarisés. Par son intermédiaire, les élèves ont ainsi accès à de nouvelles pratiques sollicitant le sensible et l'expressif. Cette conception de l'EPS bouleverse en outre la représentation de l'identité masculine de cette époque. Dominant, courageux, autoritaire et principalement reproduit par l'enseignement du sport, l'idéal masculin se trouve alors redéfini et remis en question. En dénonçant le caractère reproductif et inégalitaire du sport, les promoteurs de l'expression corporelle masculine visent non seulement à désacraliser le modèle patriarcal mais recherchent aussi l'émergence d'un nouvel idéal masculin, tourné vers plus
\end{abstract}

* Université de Strasbourg. França, Laboratoire E3S, UR 1342. E-mail: nicolas.iffrig@ hotmail.fr - https://orcid.org/0000-0001-8554-9331 E-mail: jsaintmartin@unistra.fr - https:// orcid.org/0000-0002-5880-0495 
d'émancipation et d'épanouissement. Au demeurant, les auteurs constatent l'émergence de nouvelles masculinités qu'ils qualifient de subordonnées voire marginalisées, et qui trouvent dans l'enseignement de l'expression corporelle un terrain inédit de prédilection. L'analyse exhaustive des revues professionnelles (Cahiers du GREC, Revue Esprit, Revue EP.S) et les entretiens de divers acteurs de l'expression corporelle (notamment JB. Bonange) permettent de mettre en évidence une prédominance des rapports sociaux de classe sur les rapports sociaux de genre, dans la mesure où l'expression corporelle émerge essentiellement en France dans les milieux favorisés tels que les universités, et ne parvient pas à se diffuser dans les milieux populaires, lieux de résistances d'une masculinité hégémonique traditionnelle.

Mots Clés : Expression corporelle. Éducation Physique Identités masculines. France.

\section{RESUMO}

No coração da França dos anos 1970, momento marcado por uma onda de convulsões ideológicas e culturais, a expressão corporal constitui uma experiência inovadora e única na Educação Física diante da hegemonia do esporte na área. Através de sua abordagem desregulamentada, pessoal e emancipatória, a expressão corporal marca uma ruptura na gestão do corpo das crianças em idade escolar. Por meio dela, os alunos tiveram acesso a novas práticas envolvendo o sensível e o expressivo. Essa concepção de Educação Física também afetou a representação da identidade masculina desse período. Dominante, corajoso, autoritário e principalmente reproduzido pelo ensino do esporte, o ideal masculino foi então redefinido e questionado. Ao denunciar o caráter reprodutivo e desigual do esporte, os promotores da expressão corporal masculina objetivam não apenas dessacralizar o modelo patriarcal, mas também buscar a emergência de um novo ideal masculino, voltado para mais emancipação e realização pessoal. Além disso, os autores notam o surgimento de novas masculinidades que eles qualificaram como subordinadas ou mesmo marginalizadas, e que encontram no ensino da expressão corporal um terreno inédito de predileção. A análise exaustiva de periódicos profissionais (Cahiers du GREC, Revue Esprit, Revue EP.S) e as entrevistas de vários atores da expressão corporal (em particular JB. Bonange) tornam possível destacar uma predominância das relações de classe social sobre as de gênero, na medida em que a expressão corporal surge principalmente na França em círculos privilegiados como as universidades, e não consegue disseminar nos círculos populares, locais de resistência de uma masculinidade hegemônica tradicional.

Palavras-chave: Expressão corporal. Educação Física. Identidades masculinas. França. 


\begin{abstract}
In the heart of France in the 1970s, marked by a wave of ideological and cultural upheavals and in the face of the hegemony of sports in this school subject, body language constitutes an innovative and unique experience in Physical Education. Through its deregulated, personal and emancipatory approach, body language marks a change in school children's body management. It allows students to access new practices involving both the sensitive and the expressive. This conception of physical education also deregulates the representation of the Masculine Identity of this period. Dominant, courageous, authoritarian and mainly reproduced in the teaching of sport, the masculin ideal is hence redefined and questioned. By denouncing the reproductive and unequal nature of sport, the promoters of male body language not only aim to desecrate the patriarchal model but also seek the emergence of a new male ideal, geared towards more emancipation and fulfillment. Moreover, the authors note the emergence of new masculinities which they qualify as subordinate or even marginalized, and which find in the teaching of body expression an unprecedented field of predilection. The exhaustive analysis of professional journals (Cahiers du GREC, Revue Esprit, Revue EPS) and the interviews of various specialists in corporal expression (in particular JB. Bonange) make it possible to highlight a predominance of social class relations over social relations of gender. In France, body language emerges mainly in privileged circles such as universities, and does not succeed in spreading to popular circles, places of resistance and of traditional hegemonic masculinity.
\end{abstract}

Keywords: Body language. Physical Education. Masculine Identities. France.

\title{
Introduction
}

La France des années 1960 est encore empreinte de ses traditions, rurales et chrétiennes (BAINVILLE, 2007). La patrie du Général de Gaulle se construit autour de sa figure paternelle (JACKSON, 2019). Le modèle familial, marquée par l'autorité du père, structure la société par des rôles définis dans l'exaltation d'un intérêt collectif et national ${ }^{1}$. Ce monde, pérenne et stable pour les uns, est décrit comme sclérosé et désuet par certaines tranches de la population : «le vieux monde avec ses patries, ses monuments aux morts, ses soldats trop connus ou trop inconnus, est en voie de putréfaction » (BOUJUT, 1968, p. 23).

1 «Les Français ont besoin d'avoir l'orgueil de la France ». Charles de Gaulle, cité Dans Peyrefitte (1995). 
Les populations déclassées et contestataires, portées par « la consommation de masse et les valeurs qu'elle a véhiculées » (LIPOVETSKY, 2004, p. 29), et frappées par la crise économique naissante, se liguent afin de bouleverser ce système politique et économique qui les oppresse (CASANOVA, 1968) : les doléances visent à « attaquer partout... les cloisonnements et les ségrégations entre riches et pauvres, dirigeants et dirigés, intellectuels et manuels » (HOCTAN, 2008, p. 102), au cours d'une actualisation de la "lutte des classes » (SIMON, 1968). Entre tradition et modernité, campagne et ville, jeunes et aînés, hommes et femmes, une partie de la population française, déjà divisée, s'oppose et se soulève. Les grèves ouvrières, "le plus grand mouvement de grèves $d u$ XXè siècle» (PUDAL ; RETIÈRE, 2008, p. 209), suivies par les manifestations étudiantes, forment la "révolte de la liberté contre l'oppression étatique » (ZANCARINI-FOURNEL, 2008, p. 82) et poussent le gouvernement gaulliste au fléchissement de sa politique sociale (BANTIGNY, 2018).

Les années 1970 s'ouvrent sur des réformes juridiques nécessaires face à la fronde. L'article 6 de la loi du 4 juin 1970 enterre définitivement la "puissance paternelle » et célèbre l'autorité parentale conjointe, "mettant fin à l'infériorité juridique de la mère » (BAUBÉROT, 2011, p. 179). Les revendications féministes s'imposent dans le champ politique (ARON et al., 2018). Mais « aucune réforme ne peut adapter la vieille institution au vœu des jeunes générations qui la trouvent ringarde» (SMADJA, 2011, p. 28). La rupture avec le modèle familial paternaliste et traditionnel s'institutionnalise et paraît irrémédiable : "la voix du père a résonné pour la dernière fois » (MURRAY, 1999, p. 135). Les événements auxquels assistent les Français concluent le passage de la société dans le «postmodernisme» (LIPOVETSKY, 2004), marqué par une « idéologie individualiste hédoniste » (LIPOVETSKY, 2004, p. 31), ou la victoire du privé, de la société du désir (CLOUSCARD, 1973), du spectacle (DEBORD, 1992) et de la marchandise sur le public (DEBRAY, 1978).

Modelé par des activités traditionnelles (activités physiques athlétiques, de combat, sports collectifs), l'offre culturelle sportive demeure largement différenciée selon le sexe et les transgressions toujours rares. Là encore, la modernité amène de nouvelles activités qui modifient les rapports à la pratique, à l'environnement et à soi, par une recherche effrénée de l'épanouissement personnel. De plus, le sport, qualifié de " citadelle masculine » (TERRET, 2005) car reproducteur de la domination sociale masculine, se trouve mis en danger par cette contestation. La « contre culture » (ZANCARINI-FUREL, 2008) issue de Mai 68 promeut ces activités nouvelles et tente de les introduire à l'école à travers l'éducation physique. En ce sens, nombre de ses acteurs sont 
connus grâce à leurs innovations pédagogiques au cours des "sixties $\|^{2}$. Le mouvement du GREC (Groupe de Recherches en Expression Corporelle) à Toulouse symbolise cette recherche d'innovation pédagogique. En s'opposant concrètement au modèle sportif, il est un modèle critique et alternatif, porté par l'idéologie « soixante-huitarde » (DAMAMME et al., 2008).

En effet, le groupe mené par Jean-Bernard Bonange propose l'enseignement de l'expression corporelle aux jeunes hommes et femmes, futurs enseignants d'éducation physique ${ }^{3}$. Sa pratique mixte est transgressive dans la mesure où la séparation des sexes en EPS est toujours d'actualité : «Jusqu'à la fin des années 70, au moins, l'organisation majoritairement non mixte de l'éducation physique et sportive révèle les résistances scolaires à l'égard des mutations sociales en cours » (OTTOGALLI-MAZZACAVALLO ; LIOTARD, 2012, p. 107). Naissent alors des interrogations nouvelles, notamment quant à la confrontation entre identité masculine patriarcale et activité physique féminine : quelle est l'appropriation de l'expression corporelle par ces hommes marqués par le paternalisme et la compétition sportive ? Quels modèles de masculinités sont véhiculés et promus au sein de ces groupes contestataires et novateurs ? Subsiste-t-il des résistances de la part des enseignants et des élèves face à la diffusion de ces nouvelles pratiques féminines et autocentrées?

En France, d'une part l'introduction de l'expression corporelle à l'école est un des outils pour faire évoluer durablement l'image de l'homme et l'idéal masculin dans la société. Durant cette période, les promoteurs de l'expression corporelle, en dénonçant le caractère discriminant et la violence des activités sportives pour les femmes, aspirent à désacraliser le modèle patriarcal qui se reproduirait grâce au sport à l'école. Par la remise en question du modèle sportif et par la proposition d'alternatives, les enseignants partisans des pédagogies libertaires et partie prenante des mouvements de contestation issus de mai 68 ciblent un changement de paradigme au sein de l'école. Par l'introduction de nouvelles pratiques au sein de l'éducation physique et sportive, ces acteurs visent à influencer le législateur des textes officiels, qui régissent les pratiques en EPS et à terme, les modèles de masculinité promus par la discipline et par l'école.

D'autre part, les promoteurs masculins de l'expression corporelle (GREC de Toulouse) vont s'approprier cette pratique, dans la ligne contestataire du sport, pour favoriser l'émergence d'un nouvel idéal masculin. Ce dernier promeut un modèle plus égalitaire à l'encontre des femmes, et pose la question

2 Sur la question du sport et des activités corporelles et leur évolution dans les années 1960, se reporter à Liotard (2016).

3 Sur le GREC proprement dit, se reporter à Moralès, Travaillot et Ferez (2019). 
de l'émancipation de l'homme au regard de ces nouvelles pratiques corporelles. Cette appropriation sera originale et novatrice par rapport au modèle féminin de l'expression corporelle et va engager ce «nouvel homme » dans sa singularité, son émancipation et son plaisir.

\section{Sport et masculinité hégémonique}

Les travaux novateurs de Raewyn Connell (1995) éclairent ce concept d'identité masculine, au regard des rapports sociaux de genre. En définissant une masculinité hégémonique qui " garantit la position dominante des hommes et la subordination des femmes » (CONNELL, 2014, p. 74), l'auteur s'inscrit dans une logique culturelle d'hégémonie empruntée à Antonio Gramsci. Mais cela ne revient pas à dire que «ceux qui incarnent de manière ostensible la masculinité hégémonique sont toujours les personnes les plus puissantes. Ils peuvent être des modèles, comme des acteurs de cinéma, voire des figures imaginaires » (CONNELL, 2014, p. 74).

Ainsi, la masculinité promue au sein de la société française des années 1960, comprise telle une "forme culturellement idéalisée du corps masculin " (MCKAY ; LABERGE, 2006, p. 6) est en pleine mutation au cours de la décennie suivante. "L'image d'un corps ouvrier, masculin, puissant, disposé au travail et à la lutte, s'impose dans l'imaginaire politique »(PILLON, 2011, p. 311) et incarne la masculinité hégémonique des années 1960. Cette dernière « constitue un type de masculinité particulier, qui est momentanément en position dominante et dont les différents acteurs institutionnels ou individuels s'efforcent de maintenir le rang face à la féminité et aux autres formes de masculinité » (TERRET, 2004, p. 212). Au cours des années 1960, ce modèle de masculinité des classes sociales populaires et moyennes demeure hégémonique car il est le plus répandu quantitativement dans la population française, à travers notamment la virilité des classes ouvrières et paysannes. Cette dernière représente un modèle pour les hommes de cette époque et se démarque sensiblement du dandysme bourgeois (CARASSUS, 1990) par son utilisation nécessaire de la force physique au travail. Mais aussi parce qu'il est solidement ancré dans la société depuis de nombreuses décennies. Les jeunes «entretiennent un rapport à la masculinité qui prolonge, à bien des égards, celui des jeunes ouvriers et paysans des décennies précédentes » (BAUBÉROT, 2011, p. 186). Elle se définit parallèlement à la féminité dans un rapport de complémentarité (WALCH, 2003) au sein du couple : «le genre s'affiche de manière fortement stéréotypée 
et reflète une démarcation nette entre les sexes » (BAUBÉROT, 2011, p. 187). Durant les Sixties, la domination économique, politique et sociale de l'homme sur la femme demeure une permanence, et ce modèle est largement perpétué par la pratique sportive.

\section{Le sport, une citadelle masculine ?}

Le sport est, depuis son développement au cours de la seconde moitié du XIXè siècle, d'abord un lieu d'expression masculin : "Le sport apparaît comme le symbole même de la virilité» souligne déjà Pierre de Coubertin (1992, p. 132). «Les premiers sportifs ne pensent quasiment pas le féminin. La pratique féminine demeure d'ailleurs longtemps cantonnée, rigoureusement orientée » (COURTINE, 2011, p. 237). Cette séparation entre hommes et femmes tend à reproduire et à perpétuer les valeurs viriles du sport. Les représentations liées à la virilité des années 1960 se propagent par l'exaltation de valeurs guerrières à travers la compétition et la performance: « la vigueur autant que son application surveillée, le muscle autant que son usage moral, l'exemplification des affrontements [...] Une question s'impose au coeur de l'excellence: la virilité» (COURTINE, 2011, p. 232). C'est pourquoi Thierry Terret (2005) décrit le sport telle une « citadelle masculine » imprenable pour le « sexe faible».

Cette domination se reproduit d'autant plus qu'elle prend forme dans toute la société. La presse sportive se fait l'écho de cette hégémonie masculine : « la presse sportive française conforte les bases d'une construction sociale des relations de genres» (HUMBERT, 2005, p. 261). De plus, l'école participe de ce mouvement par l'enseignement genré de l'éducation physique et sportive 4 . Ainsi, par la valorisation du sport, les hommes cherchent à perpétuer un modèle à leur avantage : "il véhicule de manière durable et massive un système de valeurs dans lequel on retrouve les caractéristiques des groupes dominants qui l'ont institué, celles d'une bourgeoisie jeune, blanche, chrétienne, libérale et masculine » (TERRET, 2005, p. 11).

4 Par exemple, les instructions Officielles du 19 octobre 1967, qui réglementent l'enseignement de l'éducation physique dans le système éducatif français, stipulent explicitement que les sports de combat sont exclusivement réservés aux garçons alors que les différentes formes de danse sont enseignées aux jeunes filles (TERRET ; COGÉRINO ; ROGOWSKI 2007). 


\section{Dépasser le modèle traditionnel du sport}

Face à ce modèle établi, émergent des alternatives que Connell (1995) qualifie de masculinité subordonnée. "Par masculinité « subordonnée », Connell se réfère aux masculinités qui se trouvent clairement dans une position dominée, voire même opprimée. Une bonne illustration de ce modèle est celui des masculinités homosexuelles, placées au plus loin de la hiérarchie des genres où elles sont assimilées aux féminités » (TERRET, 2004, p. 231). Les années 1970 deviennent progressivement le berceau de ces nouvelles revendications libérales-libertaires (CLOUSCARD, 1973) et vont révéler la volonté des femmes, mais aussi de certains hommes, de se libérer du modèle de la masculinité hégémonique antérieure, de la modifier durablement pour une société plus respectueuse des différences.

Au cours des années 1970, l'image du corps masculin se modifie, "les vieilles images de la dureté autoritaire s'effacent » (VIGARELLO, 2004, p. 232) progressivement pour laisser place à un nouvel idéal masculin. Par la recherche de l'égalité entre les sexes, les représentations de l'homme changent et s'éloignent des modèles de masculinité traditionnelle. D'une part, «le corps masculin s'effile, s'adoucit » (VIGARELLO, 2004, p. 232). Aux causes économiques et sociales s'ajoutent la diffusion par les médias de nouveaux idéaux : "nombre de signes emprunteraient au féminin : le profil par exemple « de Beatles en blue-jeans et longs cheveux en compagnie de filles en blue-jeans et cheveux mi-longs » (VIGARELLO, 2004, p. 233). D'autre part, le corps des femmes se transforme également : "les descriptions du corps féminin ont pu gommer les formes trop sexuées dans le dernier tiers du siècle, accentuer l'effacement des hanches, cultiver la discrétion de poitrine et surtout, ce qui est plus original, déployer une évidente densité musculaire ». De ces importantes mutations vont naître une "illusion androgyne » (VIGARELLO, 2004), c'est-à-dire que, les identités de genre, du moins au niveau de leurs images et de leurs représentations, vont sensiblement se rapprocher, pour parfois se confondre : "la féminisation du muscle, la masculinisation de la minceur ne sauraient, bien évidemment, réduire les deux modèles à l'identique " (VIGARELLO, 2004, p. 233).

Les mutations des identités de sexe et de genre s'expliquent par leurs origines sociales. Les événements de Mai 68, d'origine étudiante, se meuvent en combat idéologique entre une jeunesse éprise de frivolités face au pouvoir et aux normes dominantes: "Mai 68 a bien été une révolte des sujets contre les normes, à savoir au sens de l'affirmation de l'individualité contre la prétention des normes à l'universalité » (FERRY ; RENAUT, 1985, p. 121). Au cœur de 
cette lutte générationnelle s'immiscent des doléances de plusieurs ordres. Elles s'avèrent suivies d'évolutions pour le statut du «deuxième sexe » (BEAUVOIR, 2008). Une extension des droits des femmes prend effet au cours de la décennie, amorcée il est vrai dès 1967 à travers la loi Neuwirth autorisant la contraception, suivie de la dépénalisation de l'avortement par la loi Veil du 17 janvier 1975.

Cette évolution de la société française n'est pas sans conséquence sur les rapports sociaux de sexe. En effet, cette série de réformes juridiques entraîne des bouleversements sociaux majeurs, notamment de la représentation traditionnelle du couple : "le couple n'a plus pour fonction la reproduction sociale, mais la confirmation de l'identité personnelle » (WALCH, 2003, p. 206). L'accès au travail salarié et tertiarisé des femmes, où le passage de la domination du mari à celle du patron, participe au relatif rééquilibrage des forces au sein du couple, l'homme tirant jusqu'alors sa domination du travail et de son autonomie financière. Enfin, la diminution des tâches laborieuses par l'automatisation à l'usine et au champ et par la tertiarisation de l'emploi a une influence irrémédiable sur son aspect physique : "pour la paysannerie et le monde ouvrier, le statut d'homme est intimement lié à l'emploi de la force dans le cadre d'une activité productive » (COURTINE, 2011, p. 176).

Ainsi, d'une construction identitaire différenciée avant 1960 à des modèles hégémoniques de genre se confondant dans les années 1970, les rapports sociaux de sexe, et donc les identités de genre, se retrouvent remises en cause. De ces profonds changements sociétaux vont naître de nouvelles pratiques, notamment corporelles.

\section{L'émergence de l'expression corporelle}

Grâce au "régime d'égalité et ses effets » (COURTINE, 2011, p. 249) au cœur des années 1970, les femmes parviennent à atteindre des activités jusqu'alors proscrites (ARNAUD ; TERRET, 1996). En découle le développement de nouvelles valeurs, tournées vers un désir d'épanouissement personnel et la promotion d'un nouveau rapport au corps : «Pour qu'apparaisse l'envolée des frivolités, il a fallu une révolution dans la représentation des personnes et dans le sentiment de soi, bouleversant les mentalités et valeurs traditionnelles » (LYPOVETSKY, 1987, p. 67-68). Ces nouvelles préoccupations poussent à l'émergence de nouvelles pratiques, issus d'environnements où la conscience de soi et l'individualisme sont déjà développés dans la société : "L'engouement pour les thématiques du corps et de sa libération entraîne au début des années 
soixante dix la multiplication de nouvelles pratiques corporelles importées des Etats-Unis » (TRAVAILLOT, 1998, p. 46). Par l'émergence de ces activités, dites "pratiques californiennes », c'est la contestation du modèle sportif, du modèle patriarcal et plus généralement de la société qui est en jeu : « Le but de toutes ces pratiques est d'aider, grâce à un travail sur le corps, les individus névrosés par la société à retrouver normalité et santé, la définition de cette dernière tendant à s'élargir et à se rapprocher du bien être et du plaisir » (TRAVAILLOT, 1998, p. 47).

Dans ce contexte, l'expression corporelle répond à ces attentes. Décrit comme «n'étant pas une technique en soi, mais une réflexion, une pratique entre le mime, le yoga, le psychodrame et la spontanéité personnelle 》(VIGARELLO, 1982, p. 71), cette activité s'oppose au « corps conçu uniquement comme instrument » (TONELLA, 1974, p. 2) et s'inscrit dans une perspective holiste et reichienne, associant « le vivant, le mouvement [...] et l'émotion» (BERNARD, 1978, p. 110). Grâce à l'expression corporelle toute personne peut alors éprouver, laisser advenir le mouvement, rompre avec les techniques préétablies, faire que de cette cassure puisse émerger un individu neuf. Selon G. Vigarello, l'expression corporelle permet ainsi de « Jouer ses angoisses, ses peurs, ses non dit, parler avec son corps » (VIGARELLO, 1982, p. 71). Cette définition nous permet de comprendre à la fois la nouveauté mais aussi la complexité de cette activité corporelle qui émerge au cours des années 1960 et 1970 en France. D'un corps objet de performance et de mesure dans les années 1960, la décennie suivante laisse place à un corps sujet, c'est à dire non plus le moyen mais la finalité de l'activité physique : "les sports qui sont le plus souvent pratiqués dans les classes supérieures... ont d'abord pour fonction de maintenir l'individu en forme » (BOLTANSKI, 1971, p. 224).

Le courant de l'expression corporelle s'oppose donc d'une certaine manière au monde sportif compétitif en revendiquant une conception alternative du corps. Selon Guy Tonella (1974, p. 2), «le corps conçu uniquement comme instrument, sans compter avec le corps imagé, imagé et imaginant, le corps comme centre d'émergence d'une activité créatrice et assimilatrice». Il s'agit ainsi de proposer une alternative au modèle dominant, sportif et masculin. L'étude menée par Gil Mons au sein de l'université de Strasbourg en 1983 confirme les représentations ${ }^{5}$ liées à cette pratique : "l'expression corporelle est inoffensive car référée à un fondement culturel féminin qui régule le code local et la violence individuelle » (MONS, 1983, p. 57).

5 «Une observation plus synthétique permet de repérer cinq relations stables entre les représentations. Ainsi se trouvent liées : [...] la prospective, la féminité et la pensée » (MONS, 1983, p. 55). 
Par ailleurs, l'expansion des sciences cognitives (Piaget, Wallon, Schmidt) au cours de cette période vont pousser à considérer davantage les émotions comme partie intégrante du développement (WALLON, 1963) de l'individu. Selon L. Boltansky (1971, p. 224), « les femmes paraissent plus attentives que les hommes à leurs sensations morbides, s'écoutent plus que les hommes, à la façon dont les membres des classes supérieures s'écoutent plus volontiers que les membres des classes populaires ». En éducation physique et sportive, le développement de l'expression corporelle touche en premier lieu certaines universités, et en particulier l'Unité d'Enseignement et de Recherche en EPS (UEREPS) de Toulouse où Jean-Bernard Bonange crée le Groupe de Recherches en Expression Corporelle, mais aussi à l'UER des Sciences Humaines Cliniques de Paris VII où enseigne Claude Pujade-Renaud (FERREZ, 2005). Cette nouvelle forme de pratique corporelle, véritable alternative aux formes sportives alors dominantes de cette époque, interroge ouvertement le modèle de masculinité soutenu et promu : "L'impulsion vient de Jean-Bernard Bonange, professeur d'éducation physique masculin: le fait $n$ 'est pas sans importance, dans la mesure où, trop souvent, suivant le modèle socio-culturel dominant, l'expression corporelle est indexée aux activités dites féminines » (PUJADE-RENAUD, 1974, p. 75).

Entre 1967 et 1985, les promoteurs de l'expression corporelle tentent alors de s'affranchir des contenus scolaires considérés comme « des normes corporelles prônées par l'institution »(LEMONNIER, 2014, p.138) : "L'éducation physique s'efforce de cerner, circonscrire le corps, d'estimer son pouvoir productif. La danse et l'expression corporelle parlent d'un corps qui est toujours ailleurs » (PUJADE-RENAUD, 1974, p. 85). Dans les faits, sa pratique par les femmes s'apparente à la danse, qui « conserve une frange commune avec l'expression corporelle » (PUJADE-RENAUD, 1974, p. 72). Néanmoins, cette dernière " tend beaucoup plus à glisser vers l'informel " notamment parce que les pratiquantes recherchent une "motricité porteuse de signification, d'affectivité, de communication » (PUJADE-RENAUD, 1974, p. 79). Selon Jean-Louis Blein, un des membres fondateurs du GREC, elles font alors appel à leur « habitus » (BOURDIEU, 1980a) technique construit au sein de l'éducation physique démixée : «Les filles avaient souvent un passé de technique de danse » (IFFRIG, 2015, p. 99) ${ }^{6}$. Les élèves formés au sein de l'UER des Sciences Humaines Cliniques de Paris VII par Claude Pujade-Renaud sont " de très bons techniciens au niveau de l'expressivité 
$d u$ corps » (IFFRIG, 2015, p. 83) 7 allant jusqu'à viser des «formes d'activités sociales d'expression corporelle de haut niveau» (IFFRIG, 2015, p. 112).

D'une pratique féminine de l'expression corporelle influencée par le bagage culturel de ses promoteurs résulte la question de l'appropriation masculine de cette discipline, dans la mesure où les hommes ne rencontrent pas, à cette époque, l'activité danse dans le cadre scolaire.

\section{Jean-Bernard Bonange et l'émergence de l'expression corporelle masculine}

« Je crois que le genre de l'enseignant a joué. J'avais pas le même bagage culturel par rapport à la danse. Moi j'étais d'origine théâtrale, de l'improvisation, donc je dirais que c'est moins le genre que la culture dont on était porteur. Même si cette culture dépendait du genre! (IFFRIG, 2015, p. 83-84). Par cette observation, Jean-Bernard Bonange confirme la spécificité d'une expression corporelle masculine. Si la pratique féminine se construit à partir d'un habitus influencé par la danse, les hommes puisent également dans leur culture et leur formation afin de s'approprier l'expression corporelle et la faire correspondre à d'autres ambitions.

Au sein du Groupe de Recherches en Expression Corporelle de Toulouse, les hommes s'intéressent donc à cette activité dans le but de proposer un modèle sportif alternatif. Ainsi, ils fondent leur pratique à partir de ce qu'ils connaissent, c'est-à-dire leur propre culture. Cette dernière n'étant pas liée à la danse, pratique connotée féminine, leur langage technique s'avère moins développé. Ce manque de techniques corporelles expressives va alors être compensé par l'utilisation d'intermédiaires : "nous on était sans doute beaucoup moins technique mais plus créatif, au sens de l'innovation, on utilisait des supports, des matériaux, des scénographies, on passait plus par l'improvisation!" (IFFRIG, 2015, p. 83) ${ }^{9}$. Cette stratégie leur permet ainsi de se démarquer du courant de Claude Pujade-Renaud qui privilégie plutôt l'usage de médiations telles que les objets et les thèmes, l'intervention décentrée de l'enseignant en retrait de la séance, la dynamique de groupe, la pédagogie ouverte, "l'absence de technique ou l'accès à un corps langage et l'absence de capitalisation

7 Entretien avec Jean-Bernard Bonange réalisé le 21 février 2015. Dans Iffrig, N. (2015). 8 Entretien avec Michèle Baffalio-Delacroix réalisé le 13 mars 2015. Dans Iffrig, N. (2015). 9 Entretien avec Jean-Bernard Bonange. 
d'un savoir » (FEREZ, 2005, p. 57), autant d'éléments fondant l'originalité du courant toulousain. De plus, la dimension comique et burlesque, très peu présente dans l'expression corporelle féminine, s'ajoute comme un apanage masculin : "On introduisait un corps comique et burlesque qui était plus masculin que féminin »(IFFRIG, 2015, p. 84) ${ }^{10}$. Cette caractéristique est confirmée par plusieurs enseignants : "En fait les garçons étaient, en général, plus créatifs plus drôles que les filles car ils n'étaient pas empêtrés dans des considérations esthétisantes 》 (IFFRIG, 2015, p. 102-108) ${ }^{11}$. S'il se définit vierge d'antécédents liés à la danse, l'habitus des promoteurs masculins de l'expression corporelle puise alors ses références dans "la culture des colonies de vacances, où les grands jeux collectifs, et les sketchs, les scènes, les chants, sont des éléments qui ont contribué au fait que je me suis tourné vers les activités artistiques, de jeux et d'expression, de théâtre » (IFFRIG, 2015, p. 76) ${ }^{12}$.

Cette appropriation masculine d'une activité connotée féminine impose une redéfinition de l'idéal masculin promu. Jusqu'alors limité à la force physique, la robustesse, le courage, le genre masculin va sensiblement se pourvoir de caractéristiques jusqu'ici privilégiées par les femmes. Selon le sociologue, Christian Pociello (1982, p. 20), " on assiste donc, [...] au développement d'un phénomène surprenant, mais qui n'est pourtant pas totalement nouveau : la grâce masculine ». Sans reprendre explicitement cette expression de grâce masculine, J-B. Bonange confirmer toutefois que «l'expression corporelle permet l'affirmation du masculin mais aussi un monde beaucoup plus sensuel, leur part féminine tu vois. Parce que la sensibilité que l'on attribue à la féminité est évidemment présente dans le corps masculin» (IFFRIG, 2015, p. 84) ${ }^{13}$. L'expression corporelle masculine permettrait ainsi de diversifier la motricité des hommes qui était jusqu'au mitan des années 1960 cantonnée à la technique sportive et à la reproduction la plus fidèle de cette dernière. Avec cette pratique corporelle alternative qui bouleverse les codes sociaux et les représentations collectives, se pose alors la question de la hiérarchisation des modèles masculins post soixante-huitards comme en atteste le témoignage suivant d'un des membres du GREC, Guy Tonella : "Je pense que ce type d'activité interroge le genre masculin dans le sens où elle ouvre la possibilité à l'expression multiple et complexe du genre, jusqu'à la frontière ténue où le genre masculin se différencie du genre féminin » (IFFRIG, 2015, p. 93) ${ }^{14}$.

10 Entretien avec JB. Bonange.

11 Entretien avec Patricia Vidil-Grenier réalisé le 2 juin 2015. Dans Iffrig, N. (2015).

12 Entretien avec Patricia Vidil-Grenier réalisé le 2 juin 2015. Dans Iffrig, N. (2015).

13 Entretien avec JB. Bonange.

14 Entretien avec Guy Tonella réalisé le 2 mai 2015. Dans Iffrig, N. (2015). 
Au tournant des années 1970, la pratique de l'expression corporelle facilite donc en France l'émergence d'une nouvelle masculinité, éloignée du modèle hégémonique perpétué par le sport de compétition, mais aussi distinct du modèle féminin : "un double démarquage vis-à-vis des gestuels propres aux sports les plus popularisés et vis-à-vis des gestuels réputés les plus efféminés » (POCIELLO, 1982, p. 20). Les expériences pédagogiques du GREC de Toulouse ont donc favorisé l'émancipation de nouvelles caractéristiques et identités masculines par l'exploration d'une masculinité plus personnelle, dans une perspective centrée sur 1 'épanouissement de soi.

\section{Faire face à de nombreuses résistances...}

De la distinction d'une expression corporelle masculine doit naître une nuance sociologique fondamentale. En effet, l'objectif des membres du GREC de Toulouse est de démocratiser sa pratique à l'ensemble des élèves alors scolarisés dans le système éducatif français au lendemain de Mai 68. Idée elle aussi partagée par une enseignante d'expression corporelle, Michèle Baffalio-Delacroix et qui souligne cet élan en faveur de la démocratisation de nouvelles pratiques, à travers les stages, les colloques, les rencontres entre enseignants d'EPS, organisés par la Fédération Sportive et Gymnique du Travail, pourtant fortement attachée à la tradition sportive de l'éducation physique (GOIRAND et al., 2005) : «La FSGT a toujours eu le souci de la démocratisation de la pratique sportive et de la recherche de nouvelles formes de pratiques. Elle souhaitait répondre aux demandes des travailleurs et de leur famille» (IFFRIG, 2015, p. 112) ${ }^{15}$.

Néanmoins, le remplacement de l'expression corporelle par la danse dans les textes officiels de l'enseignement de l'EPS dans les collèges français de 1985 (LEMONNIER, 2014) sanctionne l'échec de la promotion et de la scolarisation de l'expression corporelle masculine à l'Ecole. Parmi les interprétations possibles, celle s'appuyant sur une approche sociologique du corps apparaît la plus probable. En effet, selon D. le Breton (2012), l'implantation d'une pratique physique ou sportive dépend avant tout du rapport au corps de la classe sociale visée. Selon lui, « un sport a d'autant plus de chances d'être adopté par les membres d'une classe sociale qu'il ne contredit pas le rapport au corps dans ce qu'il a de plus profond et de plus

15 Entretien avec M. Baffalio-Delacroix. 
profondément inconscient » Bourdieu (1980b, p. 240). En d'autres termes, il s'agit de construire puis de renforcer un rapport d'affinité entre les membres d'une classe sociale et certaines pratiques corporelles. Pour Blouin le Baron (1982, p. 62), tel semble être pourtant le cas au début des années 1980. Pour lui, « l'expression corporelle porte la marque emblématique du groupe social qui en constitue le noyau expressif ». L'expression corporelle constitue ainsi une activité physique adaptée aux usages du corps des classes favorisées, mais qui peine à s'imposer aux classes populaires, tant elles sont marquées par la valorisation de la différence des sexes, notamment l'exaltation de la force et du courage pour les garçons, dans les activités athlétiques ou les sports collectifs.

Par ailleurs, les difficultés éprouvées pour démocratiser l'expression corporelle sont également d'ordre idéologique. Les nouvelles modalités de pratique, tournées vers le ludisme, bousculent les conceptions éducatives : « Du grimper de corde on en faisait du balancer de corde style Tarzan (rires). Le peu de pratique que j'ai eu dans le secondaire j'ai essayé d'être cohérent, mais j'étais observé de la part des collègues (rires) » (IFFRIG, 2015, p. 88) ${ }^{16}$. Cette vision détournée et ludique des pratiques physiques ne sied guère à la rigidité de l'institution scolaire où les enseignants ne peuvent pas faire ce qu'ils veulent (SAINT MARTIN ; TERRET, 2019): «Il y a eu de vraies résistances. Ça a déstabilisé les conceptions, l'idéologie dominante de l'EP. Il y a une peur du désordre en EP, on est d'origine militaire... Quoi qu'on veuille, on est travaillé par une certaine morale et une certaine conception du corps qui doit être performant $\gg$ (IFFRIG, 2015, p. 88) ${ }^{17}$. D'autant que l'expression corporelle se prête difficilement aux normes scolaires : «Une méfiance persiste à l'égard de l'expression corporelle : suspecte car se prêtant mal à une programmation et une évaluation. Inquiétante car mettant en scène l'imaginaire et un corps sensoriel, pulsionnel que l'éducation physique tend précisément à neutraliser » (PUJADE-RENAUD, 1982, p. 82).

Cette critique de l'éducation physique par les promoteurs de l'expression corporelle se place dans le mouvement plus général de la dénonciation du sport et de ses pédagogies (LIOTARD, 2000), et souligne l'ambivalence entre les finalités universelles et éducatives de l'éducation physique et les activités proposées, sportives, basées sur la compétition : «il est tout à fait illusoire d'assigner à l'éducation physique une finalité purement humaniste, la recherche de l'épanouissement total d'un homme libre et créateur, alors que les moyens théoriques et pratiques dont elle dispose, relèvent d'une société dont les

16 Entretien avec JB. Bonange.

17 Entretien avec JB. Bonange. 
impératifs sont dictés par des intérêts qui ne vont pas nécessairement dans le même sens »(BERNARD, 1975, p. 728). Cette ambiguité souligne la présence de deux conceptions éducatives et idéologiques en tension.

À ces difficultés viennent s'agréger de nouvelles résistances, notamment pour ce qui concerne les valeurs véhiculées par l'expression corporelle et les revendications étudiantes qui se réclament ouvertement des mouvements contestataires : "Indéniablement, le GREC est né dans un courant post soixante huitard, porté pas des aspirations libertaires. Un désir à la fois de lutter contre les diverses pressions et injustices sociales et de trouver une liberté et un épanouissement de l'individu » (IFFRIG, 2015, p. 98) ${ }^{18}$. Si ces acteurs appartiennent et participent à ces mouvements contestataires, leurs idéaux politiques infusent leurs pratiques d'enseignants : "Il me semble que les influences idéologiques les plus évidentes avaient à voir d'une part avec une position politique de gauche, voire d'extrême gauche, et, d'autre part avec une attirance vers tout ce qui était anti-sportif et plutôt tourné vers les activités psychomotrices et créatrices » (IFFRIG, 2015, p. 92).

L'analyse politique (GLEYSE, 2004) de ces clivages permet ainsi de définir deux grands mouvances idéologiques. Ici naissent les principales divergences entre un rapport au corps « de gauche » voire « d'extrême gauche », favorisant l'émancipation de chacun au sein d'une masculinité choisie et voulue, promue par les concepteurs de l'expression corporelle ${ }^{19}$, et un rapport au corps « de droite», visant une masculinité patriarcale, performante et compétitive et défendue par le pouvoir en place, notamment par le gouvernement du Général de Gaulle avant sa démission du pouvoir en 1969.

\section{Conclusion}

L'analyse des masculinités promues ou réprimées par les différents acteurs de l'expression corporelle nous permet de définir en France différents enjeux idéologiques qui traversent l'enseignement de l'EPS dans l'institution scolaire. D'une analyse de prime abord sexuée, le présent travail de recherche cherche à démontrer la prédominance des rapports sociaux de classe et de sexe dans la définition d'une culture corporelle dispensée au sein des institutions

18 Entretien avec JL. Blein.

19 Sur cette question se reporter notamment au numéro spécial de la revue STAPS, Mai $68, \mathrm{n}^{\circ} 126,2019$. 
éducatives françaises entre 1967 et 1985. Malgré l'originalité d'un discours positiviste et émancipateur, les promoteurs de l'expression corporelle masculine se heurtent en effet, dans leur projet de diffusion de cette pratique alternative aux pratiques sportives de compétition, à des résistances liées à l'existence d'une masculinité hégémonique qui laisse à la fois dans les discours et dans les pratiques professionnelles des enseignants d'EPS peu de place aux masculinités subordonnées. Ces acteurs, tous membres du GREC de Toulouse, doivent ainsi faire face à de nombreuses difficultés et obstacles qui rendent difficiles, sinon impossibles la remise en cause de cet idéal masculin dominant, érigé en France comme un modèle hégémonique depuis la Seconde Guerre mondiale. En outre, la puissance des normes et usages de l'institution scolaire, au demeurant bien étudiée par P. Arnaud (1989)à travers son modèle d'analyse de l'orthodoxie scolaire, gène considérablement leurs volontés d'innovation et de changement en EPS alors que l'on constate par ailleurs la timide intégration de la danse dans les programmes scolaires de l'EPS des collèges en 1985 (OTTOGALLIMAZZACAVALLO ; LIOTARD, 2012). Dans ces conditions, la diffusion de l'expression corporelle masculine reste dépendante du décalage entre le discours libéral-libertaire promu par ses acteurs, et les représentations liées à l'idéal masculin populaire et hégémonique incarné par la place dominante occupée par le sport et ses formes de pratiques traditionnelles. Néanmoins, les effets de Mai 68 commencent à se faire entendre lorsqu'il s'agit de réfléchir à la place du corps dans les apprentissages des élèves, surtout lorsque ces derniers sont en situation de difficulté scolaire. À l'orée des années 1980, force est de constater les relations de plus en plus explicites entre culture corporelle de classe et logique de l'activité corporelle. Nous pourrions alors prolonger nos investigations pour démontrer l'importance de l'habitus sur les pratiques physiques et sportives des individus et sur les rapports sociaux de genre et nuancer l'importance de la lecture genrée en éducation physique et à l'Ecole de la République française qui est par ailleurs traversée par de nombreux débats idéologiques (ROBERT, 2015).

\section{RÉFÉRENCES}

ARON, Jean-Paul et al.(org.) Commémorer Mai 68, Paris : Gallimard, 2018.

ARNAUD, Pierre. Contribution à une histoire des disciplines d'enseignement : la mise en forme scolaire de l'éducation physique. Revue française de pédagogie, [s.l.], n. 89, p. 29-34, 1989. 
ARNAUD, Pierre ; TERRET, Thierry. Histoire du sport féminin. Paris : L'Harmattan, 1996.

BAINVILLE, Jacques. Histoire de France (1924). Paris : Tallandier, 2007.

BANTIGNY, Ludivine. 1968 : De grands soirs en petits matins. Paris : Seuil, 2018.

BAUBEROT, Arnaud. On ne naît pas viril, on le devient. In : CORBIN, Alain ; VIGARELLO, Georges ; COURTINE, Jean-Jacques (org.). Histoire de la virilité: La virilité en crise ? Le XXè - XXIè siècle. Paris : Seuil, 2011. p. 159-184.

BEAUVOIR, Simone. Le deuxième sexe. Paris : Gallimard, 2008.

BERNARD, Michel. L'ambivalence du corps. Esprit, [s.l.], n. 446, v. 5, p. 724-738, 1975.

BERNARD, Michel. Quelles pratiques corporelles maintenant? Paris : Corps \& culture, 1978.

BLOUIN LE BARON, Jacqueline. L'expression corporelle. Revue EPS, Paris, n. 178, p. 58-62, 1982.

BOLTANSKI, Luc. Les usages sociaux du corps. Annales. Economies, Sociétés, Civilisations, [s.l.], n.1, p. 205-233, 1971.

BOUJUT, Pierre. Réveillez-vous ! Ou la révolte contre le désespoir. La Tour de feu, Paris, n. 98, p. 21-34, juil./sept. 1968.

BOURDIEU, Pierre. Questions de sociologie. Paris : Editions de Minuit, 1980a.

BOURDIEU, Pierre. La distinction. Paris : Edition Minuit, 1980b.

CARASSUS, Emilien. Dandysme et aristocratie. Romantisme, [s.l.], v. 20, n. 70, p. 25-37, 1990.

CASANOVA, Antoine. Ouvriers, intellectuels et étudiants. La Nouvelle Critique, Paris, 3 juin 1968. (Numéro Spécial).

CLOUSCARD, Michel. Néofascisme et idéologie du désir. Paris : Gonthier, 1973.

CONNELL, Raewyn Connell. Masculinities. Berkeley : University of California Press, 1995.

CONNELL, Raewyn Connell. Masculinités : Enjeux sociaux de l'hégémonie. Paris : Editions Amsterdam, 2014.

COUBERTIN, Pierre. Essais de psychologie sportive. Grenoble : Jérôme Millon, 1992.

COURTINE, Jean-Jacques. Impossible virilité. In : CORBIN, Alain ; VIGARELLO, Georges ; COURTINE, Jean-Jacques (org.). Histoire de la virilité. Tome 3. Paris : Editions Seuil, 2011. p. 8-11.

DAMAMME, Dominique et al. Mai Juin 68. Paris : Les éditions de l'Atelier, 2008.

DEBORD, Guy. La société du spectacle. Paris : Gallimard, 1992. 
DEBRAY, Régis. Modeste contribution aux cérémonies du dixième anniversaire. Paris : Maspero, 1978.

FEREZ, Sylvain. Mensonge et vérité des corps en mouvement: L'œuvre de Claude Pujade-Renaud. Paris : L'Harmattan, 2005.

FERRY, Luc ; RENAUT, Alain. La pensée 68 : Essai sur l'anti-humanisme contemporain. Paris : Gallimard, 1985.

GLEYSE, Jacques. La gauche et la droite en éducation physique en France au XXè siècle. In : LECOQ, Gilles ; GLEYSE, Jacques ; CEBE, Didier. L'EPS : de ses environnements à l'élève. Paris : Vigot, 2004. p. 357-443.

GOIRAND, Paul et. al. Les stages Maurice Baquet 1965-1975 : Genèse du sport de l'enfant, Paris : L'Harmattan, 2005.

HOCTAN, Caroline. Mai 68 en revues. Paris : Imec 2008. (Collection Pièces D’Archives).

HUMBERT, Henri. La presse sportive française et l'encouragement à l'hégémonie masculine (1900-1970). In : TERRET, Thierry ; LIOTARD, Philippe. Sport et Genre. Excellence féminine et masculinité hégémonique, 2005. v. 2, p. 241-262.

IFFRIG, Nicolas. Virilité et expression corporelle en EPS de 1967 à 1985. Mémoire de Master. Strasbourg : Université de Strasbourg, 2015.

JACKSON, Julian. De Gaulle : une certaine idée de la France, Paris : Seuil, 2019.

LE BRETON, David. La sociologie du corps. Paris : PUF, 2012.

LEMONNIER, Jean-Marc. L'indétermination de la danse à l'école. In : ATTALI, Michaël ; SAINT-MARTIN, Jean (org.). A l'école du sport : Epistémologie des savoirs corporels du XIXè siècle à nos jours. Paris : De Boeckm 2014.

LIOTARD, Philippe. Compréhension du corps et dénonciation du sport (1968-1979). In : TERRET, Thierry (org.). Éducation physique, Sport et loisirs 1970-2000. Paris : Editions AFRAPS ; Chamalières, 2000. p. 121-138.

LIOTARD, Philippe (org.). Le sport dans les sixties : pratiques, valeurs, acteurs. Reims : EPURE, 2016.

LIPOVETSKY, Gilles. L'emprise de l'éphémère : La mode et son destin dans les sociétés modernes. Paris : Gallimard, 1987.

LIPOVETSKY, Gilles. Les temps hypermodernes. Paris : Grasset, 2004.

MCKAY, Jim ; LABERGE, Suzanne. Sport et masculinités. Clio. Femmes, genre, histoire, [s.l.], n. 23, p. 239-267, 2006.

MONS, Gil. De l'expression corporelle aux transfigurations. Maîtrise en STAPS. Strasbourg : Université de Strasbourg, 1983. 
MORALÈS, Yves ; TRAVAILLOT, Yves ; FEREZ, Sylvain. Le GREC (1968-1976), une contestation «par corps» de la formation des enseignants d'éducation physique et sportive. Sciences sociales et sport, [s.l.], v. 1, n. 13, p. 57-83, 2019.

MURAY, Philippe. Après l'Histoire. Paris : Les Belles-Lettres, 1999.

OTTOGALLI-MAZZACAVALLO, Cécile ; LIOTARD, Philippe. L'apprentissage du genre en éducation physique. Devenir homme ou femme par l'exercice. L'éducation du corps à l'école. Mouvements, normes et pédagogies (1881-2011), [ s.l.], p. 93-113, 2012.

PEYREFITTE, Alain. C'était de Gaulle. Tome I. Paris : Fayard, 1995.

PILLON, Thierry. Virilité ouvrière. In : CORBIN, Alain ; VIGARELLO, Georges ; COURTINE, Jean-Jacques (org.). Histoire de la virilité. Paris : Seuil, 2011. v. 3, p. 303-325.

POCIELLO, Christian. Sportifs glisseurs et sportifs rugueux. Dans Le corps... entre illusions et savoirs. Esprit, [s. l.], n. 2, p. 20, p. 19-33, 1982.

PUDAL, Bernard ; RETIERE, Jean-Noël. Les grèves ouvrières de 68, un mouvement social sans lendemain mémoriel. In : DAMAMME, Dominique ; GOBILLE, Boris ; MATONTI, Frédérique ; PUDAL, Bernard. Mai Juin 68. Paris : Les éditions de l'Atelier, 2008. p. 207-221.

PUJADE-RENAUD, Claude. Expression corporelle : langage du silence. Paris : Éditions sociales françaises, 1974.

ROBERT, André Désiré. L'École en France de 1945 à nos jours. Grenoble : PUG, 2015.

SAINT-MARTIN, Jean ; TERRET, Thierry (org.). Pierre Arnaud : Historien de 1'EPS et du sport scolaire, Paris : L'Harmattan, 2019.

SIMON, Michel. Mai-Juin 68, deux mois de luttes de classes en France. La Nouvelle Critique, Paris, n. 197, p. 2-9, 1968.

SMADJA, Éric. Le couple et son histoire. Paris : PUF, 2011.

TERRET, Thierry. Sport et masculinité: une revue de questions. Staps, Paris, n. 4, p. 209-225, 2004.

TERRET, Thierry. La conquête d'une citadelle masculine. Sport et genre. Espaces et temps du sport. Paris : L'Harmattan, 2005. v. 1.

TERRET, Thiery ; COGÉRINO, Geneviève ; ROGOWSKI, Isabelle. Pratiques et représentations de la mixité en EPS, Revue EPS, [s.l.], v. 47, n. 4, p. 25-42, 2007.

TONELLA, Guy. Editorial. Les cahiers du GREC, n. 8-9, p. 2, 1974.

TRAVAILLOT, Yves. Sociologie des pratiques d'entretien du corps. Paris : PUF, 1998. VIGARELLO, Georges. Les vertiges de l'intime. Esprit, [s.l.], n. 62, v. 2, p. 68-78, 1982. VIGARELLO, Georges. Histoire de la beauté : Le corps et l'art d'embellir de la Renaissance à nos jours. Paris : Seuil, 2004. 
IFFRIG, N. ; SAINT-MARTIN, J. Identités masculines, expression corporelle et éducation physique...

WALCH, Agnès. Histoire du couple en France : de la Renaissance à nos jours. Paris : Ouest-France, 2003.

WALLON, Henri. Les origines de la pensée chez l'enfant. Paris : PUF, 1963.

ZANCARINI-FOURNEL, Michelle. Le moment 68 : Une histoire contestée. Paris : Seuil, 2008.

Texte reçu le 10/11/2020.

Texte approuvé le 02/03/2021. 